\title{
Cognitive versus virtual reality simulation for evaluation of technical skills in neurosurgery
}

\author{
Steven Knafo, MD, PhD, ${ }^{1}$ Nicolas Penet, MD, ${ }^{2}$ Stephan Gaillard, MD, ${ }^{3}$ and Fabrice Parker, MD${ }^{1}$ \\ 1'Department of Neurosurgery, Bicêtre Hospital, AP-HP, Université Paris-Saclay, Le Kremlin-Bicêtre; ${ }^{2}$ Department of \\ Neurosurgery, Lariboisière Hospital, AP-HP, Nord-Université de Paris; and ${ }^{3}$ Department of Neurosurgery, Foch Hospital, \\ Suresnes, France
}

OBJECTIVE Simulation is gaining momentum as a new modality of medical training, particularly in acute care settings such as surgery. In the present study, the authors aimed to compare individual cognitive skills with manual abilities as assessed by virtual reality (VR) simulation among neurosurgical residents.

METHODS Participants were asked to complete a multiple-choice questionnaire assessing their surgical abilities regarding three basic neurosurgical procedures (endoscopic third ventriculostomy, cranial meningioma, and lumbar laminectomy). They subsequently performed these same three procedures on a VR simulator (NeuroTouch).

RESULTS The authors found that cognitive scores correlated with self-evaluation of surgical experience and autonomy. On the contrary, VR simulation, as assessed by NeuroTouch automated scoring, did not reflect participants' cognitive or self-evaluation of their surgical proficiency.

CONCLUSIONS The results of this study suggest that neurosurgical education should focus as much on cognitive simulation (e.g., careful planning and critical appraisal of actual procedures) as on VR training of visuomotor skills. https://thejns.org/doi/abs/10.3171/2021.5.FOCUS201007

KEYWORDS simulation; NeuroTouch; technical skills; cognitive skills; neurosurgery

$\mathrm{W}$ ITH the advent of simulation training, two different aspects of medical practice are increasingly recognized: "technical skills," relating to the manual execution of a given procedure, and "nontechnical skills," referring to the interpersonal relationships occurring around this procedure. Simulation has been widely studied in a variety of acute care settings (including surgery and the ICU) to assess interpersonal skills, ${ }^{1}$ and many studies have demonstrated that simulation could improve communication, teamwork, and, eventually, patient outcomes in the operating room. ${ }^{2}$

Another aspect of simulation for surgery is directed toward the acquisition of procedural skills. Surgical simulators relying on virtual reality (VR), robotics, and imaging integration have recently emerged as alternatives to training in cadaveric laboratories. ${ }^{3,4}$ In neurosurgery only, several sophisticated simulators dedicated to different types of procedures (e.g., endoscopic, endovascular, and spine) are now commercially available. ${ }^{5-7}$ However, whereas many studies have investigated the role of simulation in the evaluation of interpersonal skills, very few have focused on cognitive abilities at the individual level, such as surgical planning, risk awareness, and adaptive behavior.

In the present study, we aimed to compare individual cognitive skills with manual abilities as assessed by VR simulation of similar surgical procedures among neurosurgical residents.

\section{Methods}

An observational and comparative study involving neurosurgical residents from a single regional program (Assistance Publique-Hôpitaux de Paris, France) was conducted. Residents were asked to self-evaluate their level of autonomy using a scale ranging from 1 (unable to perform the surgery even with supervision) to 10 (able to perform the surgery without supervision) and to estimate the number of procedures they had performed as the main operator.

ABBREVIATIONS ETV = endoscopic third ventriculostomy; $P G Y=$ postgraduate year; VR = virtual reality.

SUBMITTED November 26, 2020. ACCEPTED May 24, 2021.

INCLUDE WHEN CITING DOI: 10.3171/2021.5.FOCUS201007. 


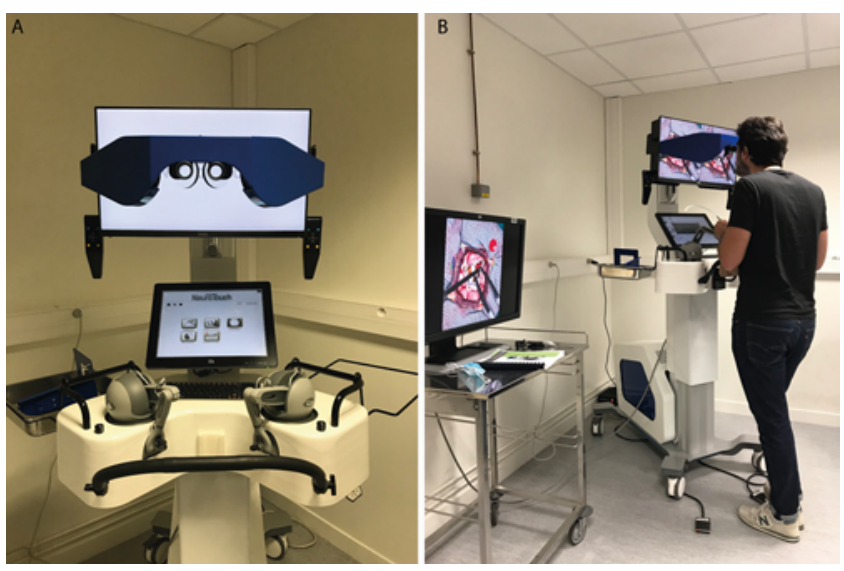

FIG. 1. A: NeuroTouch, a VR simulator for neurosurgery. B: A participant using the VR simulator to perform a virtual neurosurgical procedure.

\section{Cognitive Evaluation}

An online survey was completed by the participants (https://forms.gle/Zm9euCSf1jjRBYvz9; English translation as Supplementary Data 1). This survey included three clinical cases involving basic neurosurgical procedures: endoscopic third ventriculostomy (ETV), cranial meningioma, and lumbar stenosis. Each case consisted of a short clinical description accompanied by radiological illustrations, followed by 10 multiple-choice questions. Questions related to anatomy (identification of anatomical landmarks on operative views, and expected relationships between structures), procedure (patient installation and order of surgical steps), or decision-making (management of surgical findings, and reaction to an unexpected event). There were 10 questions for each of these three categories.

\section{VR Evaluation}

After completion of the cognitive survey, residents performed similar procedures (ETV, convexity meningioma, and hemilaminectomy) on a VR simulator (NeuroTouch, now NeuroVR, from CAE Healthcare). NeuroTouch is a commercially available VR simulator specifically designed for neurosurgery, comprising a stereoscopic view and two haptic handles (Fig. 1). ${ }^{6}$ A range of physical interchangeable tools are available (suction, bipolar forceps, ultrasonic aspirator, and microscissors) to interact with simulated tissues. Several surgical scenarios are available, and automated performance scores are given as percentages according to prespecified metrics. The automated scoring metrics for the three procedures, as described in the NeuroTouch version 15.02 courseware, are provided online at https://www.caehealthcare.com/media/files/User_Guides/ NeuroVR-User-Guide.pdf.

\section{Statistical Analysis}

Individual cognitive and manual scores were collected for each participant. To allow comparisons between students and across modalities, cognitive and manual scores were normalized and transformed as percentages. Correlations between scores and neurosurgical experience were performed using Spearman nonparametric correlations. All data were analyzed with IBM SPSS Statistics (version 20.0, IBM Corp.) software.

\section{Results}

\section{Participants}

Twelve neurosurgical residents and two senior neurosurgeons participated in the study. Two residents each were in postgraduate years (PGYs) 1,2, and 3, and 3 residents each were in PGYs 4 and 5. For ETV procedures, self-evaluation ranged from 1 (0 procedures performed, PGY-1) to 10 (> 20 procedures performed, senior surgeon), with a mean of 4.2 procedures. For meningioma procedures, self-evaluation ranged from 1 ( 0 procedures performed, PGY-1) to 10 ( $>40$ procedures performed, senior surgeon), with a mean of 6 procedures. For lumbar laminectomy procedures, self-evaluation ranged from $2(0$ procedures performed, $\mathrm{PGY}-1$ ) to 10 ( $>50$ procedures performed, senior surgeon), with a mean of 17.8 procedures (Table 1).

\section{Cognitive Evaluation Versus Self-Evaluation}

The mean cognitive scores across participants were 53/100 (range 30-95) for ETV, 54/100 (range 25-91) for meningioma, and 51/100 (range 21-100) for lumbar laminectomy. Cognitive evaluation positively correlated with self-evaluation of technical ability by the participants; the Spearman rho-coefficient between cognitive normalized score and self-evaluation was 0.8 for ETV ( $p<0.01), 0.5$ for meningioma $(\mathrm{p}=0.07)$, and 0.5 for laminectomy ( $\mathrm{p}$ $=0.05$ ). Overall, cognitive assessment strongly correlated with self-evaluation of surgical experience and autonomy (Spearman rho-coefficient $=0.7, \mathrm{p}=0.01$; Fig. 2).

\section{VR Evaluation Versus Self-Evaluation}

The mean manual scores across participants were -112 (range -260 to 0) for ETV, -52 (range -119 to 23) for meningioma, and -50 (range -137 to 37 ) for laminectomy. Manual evaluation with the NeuroTouch did not correlate with the resident's self-evaluation of their technical abilities; the Spearman rho-coefficient between manual and self-evaluation was 0.3 for ETV ( $p=0.3),-0.5$ for meningioma $(\mathrm{p}=0.1)$, and -0.1 for laminectomy $(\mathrm{p}=0.7)$. Overall, manual assessment based on the NeuroTouch automated scores did not correlate with self-evaluation of surgical proficiency $($ Spearman rho-coefficient $=0.01, \mathrm{p}=$ 0.98; Fig. 2).

\section{Cognitive Evaluation Versus VR Evaluation}

Altogether, there was no correlation between cognitive and VR evaluations of technical skills among neurosurgical residents based on individual participant scores (Spearman rho-coefficient $=-0.05, \mathrm{p}=0.8$ ).

\section{Discussion}

In our study, cognitive evaluation of technical skills using a simple clinical questionnaire correlated with the resident's experience and self-evaluation of his or her surgical proficiency. On the contrary, VR simulation with the 
TABLE 1. Participant self-evaluation of surgical autonomy and experience by procedure

\begin{tabular}{|c|c|c|c|c|c|c|c|}
\hline \multirow{2}{*}{$\begin{array}{c}\text { Participant } \\
\text { No. }\end{array}$} & \multirow[b]{2}{*}{ Experience } & \multicolumn{2}{|c|}{ ETV } & \multicolumn{2}{|c|}{ Meningioma } & \multicolumn{2}{|c|}{ Laminectomy } \\
\hline & & Experience & Autonomy & Experience & Autonomy & Experience & Autonomy \\
\hline 1 & PGY-1 & 0 & 1 & 0 & 1 & 0 & 2 \\
\hline 2 & PGY-1 & 0 & 1 & 0 & 1 & 2 & 4 \\
\hline 3 & PGY-2 & 1 & 4 & 0 & 2 & 3 & 6 \\
\hline 4 & PGY-2 & 0 & 3 & 2 & 5 & 3 & 5 \\
\hline 5 & PGY-3 & 2 & 4 & 1 & 3 & 12 & 5 \\
\hline 6 & PGY-3 & 3 & 7 & 0 & 5 & 8 & 8 \\
\hline 7 & PGY-4 & 12 & 7 & 10 & 5 & 30 & 8 \\
\hline 8 & PGY-4 & 0 & 5 & 0 & 5 & 30 & 8 \\
\hline 9 & PGY-4 & 3 & 6 & 1 & 5 & 30 & 9 \\
\hline 10 & PGY-5 & 4 & 8 & 6 & 7 & 10 & 10 \\
\hline 11 & PGY-5 & 0 & 6 & 0 & 5 & 5 & 8 \\
\hline 12 & PGY-5 & 4 & 7 & 4 & 8 & 25 & 10 \\
\hline 13 & Senior surgeon & 20 & 10 & 20 & 10 & 40 & 10 \\
\hline 14 & Senior surgeon & 10 & 10 & 40 & 10 & 50 & 10 \\
\hline
\end{tabular}

"Experience" refers to the number of procedures completed as first operator by the resident. "Autonomy" refers to selfevaluation of the resident's ability to perform this procedure alone.

NeuroTouch, as assessed by its automated scoring system, showed no correlation with experience or autonomy.

This result contradicts previous studies published by the group that designed NeuroTouch, which stated that it could discriminate between junior residents, senior residents, and board-certified neurosurgeons. ${ }^{8}{ }^{89}$ However, in these studies, only some of the advanced metrics, such as ultrasonic aspirator "path length index" or "bimanual forces ratio," correlated with the participant's surgical

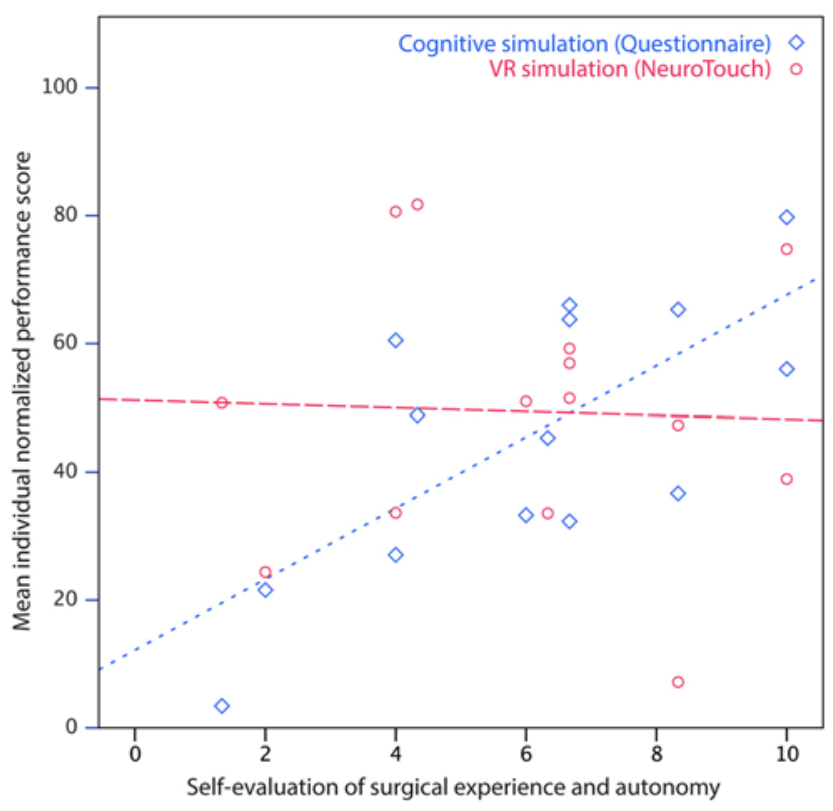

FIG. 2. Cognitive evaluation versus VR evaluation of surgical skills. experience. Another group working on a different VR simulator (ImmersiveTouch) did not demonstrate significant differences in terms of performance metrics between first-year medical students, neurosurgery applicants, and current neurosurgical residents. ${ }^{10,11}$

One explanation for such an absence of correlation between the simulator assessments and surgical experience could be that metrics need to be refined to capture the subtle progression in visuomotor performance along neurosurgical training. For instance, Roitberg et al. combined haptic perception, motor planning, and spatial memory into an individual "sensory-motor quotient" that tended to discriminate neurosurgical residents from medical students. ${ }^{10}$ Indeed, even if VR simulators fail to capture actual surgical proficiency, they can still assess some form of visuomotor performance. Such psychometric evaluation could be useful, for example, in screening applicants for a neurosurgical program. ${ }^{9}$

Another explanation for the observed discrepancy is that skills assessed by the VR simulator are obviously not the same as those measured by the questionnaire. However, our aim in this study was not to compare these two evaluation modalities but rather to compare each one with the resident's self-evaluation of their surgical experience and proficiency. Our assumption was that surgical training should have developed both cognitive and manual abilities and that, therefore, both would correlate with the participant's surgical experience.

\section{Conclusions}

Surgical proficiency relates as much to cognitive abilities as manual dexterity. In other words, performing a successful surgery requires more than knowing how to handle the instruments properly. Individual cognitive abilities such as rigorous surgical planning, anatomical 
knowledge, risk awareness, and adaptive learning are at least as important as visuomotor performance. As such, we suggest that selection and training of future surgeons should also focus on these critical cognitive abilities rather than relying solely on sophisticated, albeit limited, VR manual skills.

\section{References}

1. Armenia S, Thangamathesvaran L, Caine AD, King N, Kunac A, Merchant AM. The role of high-fidelity team-based simulation in acute care settings: a systematic review. Surg $J$ (NY). 2018;4(3):e136-e151.

2. Fleetwood VA, Veenstra B, Wojtowicz A, Kerchberger J, Velasco J. Communication through simulation: Developing a curriculum to teach interpersonal skills. Surgery. 2018; 164(4):802-809.

3. Bernardo A. Establishment of next-generation neurosurgery research and training laboratory with integrated human performance monitoring. World Neurosurg. 2017;106:991-1000.

4. Choudhury N, Gélinas-Phaneuf N, Delorme S, Del Maestro R. Fundamentals of neurosurgery: virtual reality tasks for training and evaluation of technical skills. World Neurosurg. 2013;80(5):e9-e19.

5. Barber SR, Jain S, Mooney MA, Almefty KK, Lawton MT, Son YJ, Stevens SM. Combining stereoscopic video and virtual reality simulation to maximize education in lateral skull base surgery. Otolaryngol Head Neck Surg. 2020;162(6): 922-925

6. Delorme S, Laroche D, DiRaddo R, Del Maestro RF. NeuroTouch: a physics-based virtual simulator for cranial microneurosurgery training. Neurosurgery. 2012;71(1 Suppl Operative):32-42.

7. Bernardo A. Virtual reality and simulation in neurosurgical training. World Neurosurg. 2017;106:1015-1029.

8. Alotaibi FE, AlZhrani GA, Mullah MAS, Sabbagh AJ, Azarnoush H, Winkler-Schwartz A, Del Maestro RF. Assessing bimanual performance in brain tumor resection with NeuroTouch, a virtual reality simulator. Neurosurgery. 2015;11(1) (suppl 2):89- 98.
9. Winkler-Schwartz A, Bajunaid K, Mullah MAS, Marwa I, Alotaibi FE, Fares J, et al. Bimanual psychomotor performance in neurosurgical resident applicants assessed using NeuroTouch, a virtual reality simulator. J Surg Educ. 2016; 73(6):942-953.

10. Roitberg BZ, Kania P, Luciano C, Dharmavaram N, Banerjee $\mathrm{P}$. Evaluation of sensory and motor skills in neurosurgery applicants using a virtual reality neurosurgical simulator: the sensory-motor quotient. J Surg Educ. 2015;72(6):1165-1171.

11. Roitberg B, Banerjee P, Luciano C, Matulyauskas M, Rizzi S, Kania P, Gasco J. Sensory and motor skill testing in neurosurgery applicants: a pilot study using a virtual reality haptic neurosurgical simulator. Neurosurgery. 2013;73(suppl 1): $116-121$

\section{Disclosures}

The authors report no conflict of interest concerning the materials or methods used in this study or the findings specified in this paper.

\section{Author Contributions}

Conception and design: Knafo. Acquisition of data: Knafo, Penet. Drafting the article: Knafo. Critically revising the article: Knafo, Gaillard, Parker. Reviewed submitted version of manuscript: Gaillard, Parker. Study supervision: Parker.

\section{Supplemental Information}

Online-Only Content

Supplemental material is available online.

Supplementary Data 1. https://thejns.org/doi/suppl/10.3171/ 2021.5.FOCUS201007.

\section{Correspondence}

Steven Knafo: Bicêtre Hospital, AP-HP, Université Paris-Saclay, Le Kremlin-Bicêtre, France. steven.knafo@aphp.fr. 